\title{
On the Use of High-resolution Time-frequency Distribution Based on a Polynomial Compact Support Kernel for Fault Detection in a Two-level Inverter
}

\author{
Sara Seninete ${ }^{1 *}$, Mansour Abed ${ }^{1}$, Azeddine Bendiabdellah², Malika Mimi ${ }^{1}$, Adel Belouchrani , \\ Abdelaziz Ould Ali', Bilal Djamal Eddine Cherif ${ }^{4}$

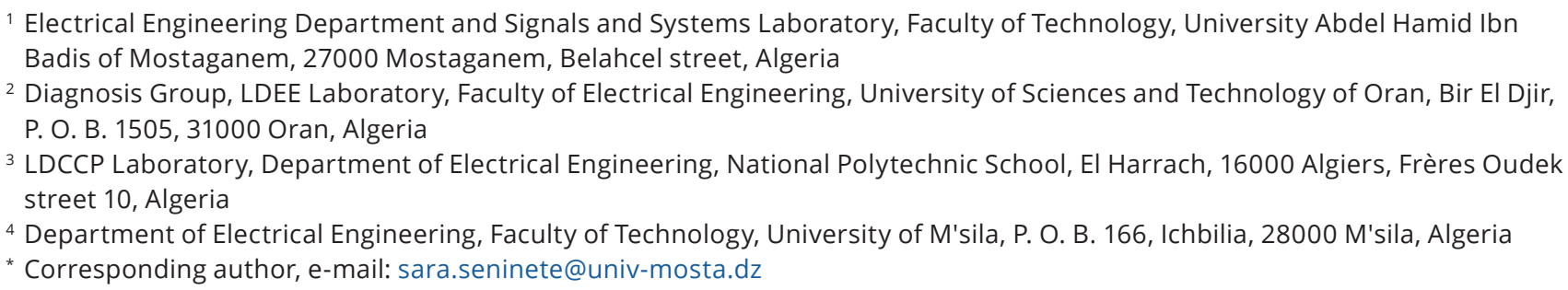

Received: 21 December 2019, Accepted: 17 March 2020, Published online: 31 August 2020

\begin{abstract}
Quadratic Time-Frequency Distributions (TFDs) become a standard tool in many fields producing nonstationary signatures. However, these representations suffer from two drawbacks: First, bad time-frequency localization of the signal's autoterms due to the unavoidable crossterms generated by the bilinear form of these distributions. This results on bad estimation of the Instantaneous Frequency (IF) laws and decreases, in our case, the ability to precisely decide the existence of a motor fault. Secondly, the TFD's parameterization is not always straightforward. This paper deals with faults' detection in two-level inverter feeding induction motors, in particular open-circuit Insulated Gate Bipolar Transistor (IGBT) faults. For this purpose, we propose the use of a recent highresolution TFD, referred as PCBD for Polynomial Cheriet-Belouchrani Distribution. The latter is adjusted using only a single integer that is automatically optimized using the Stankovic concentration measure, otherwise, no external windows are needed to perform the highest time-frequency resolution. The performance of the PCBD is compared to the best-known quadratic representations using a test bench. Experimental results show that the frequency components characterizing open-circuit faults are best detected using the PCBD thanks to its ability to suppress interferences while maintaining the signal's proper terms.
\end{abstract}

Keywords

Time-Frequency Distributions (TFDs), open-circuit fault detection, diagnosis, Polynomial Compact Support Kernel (PCSK), Stankovic measure

\section{Introduction}

Fault detection in electrical drives under nonstationary conditions has been gaining significance in recent times. This is because the induction motors used in these drives have become widely employed in most types of industrial speed control applications mainly thanks to their high reliability, low cost per power unit, high power per volume unit and very low maintenance requirement [1]. However, failures are unavoidable. In order to increase the productivity, supervise the reliability and ensure the safety of the entire installation, a necessary permanent condition monitoring of electrical drives should definitely take place to early detect and localize the presence of faults in the system.
Various researchers have focused their studies on faults' detection and localization in static converters and more specifically in three-phase power inverters fed induction motor with their essential function of speed variation [2]. Several surveys denote that about $53 \%$ of the faults in variable-speed ac drives in industry are due to failures in control circuits. $38 \%$ of these failures are in power equipments, notably power devices, capacitors [3]. Particularly, gate control circuits remain the most susceptible components $[4,5]$. The present work focuses on two-level inverter fault detection. As we mentioned above, the most critical faults in an inverter depend 
mainly on the IGBTs' control signals. In fact, two types of faults can arise on the inverter IGBT switch: a short-circuit fault or an open-circuit one $[2,6]$. The latter is considered in the present work. Most of the failures including IGBT can be detected by the drive electronics. These latter provide driving capability, optical isolation, and integrated safety. Furthermore, the gate drive optcouplers contain critical circuit isolation and short-circuit protection. Since the IGBT fault investigated in this paper is in fact an open-circuit fault, this means that the current sensed from the faulty IGBT is zero and therefore this fault cannot be detected by the drive electronics system. Otherwise, many diagnoses based on processing of stator current were proposed. For example, Fourier transform provides good results in stationary conditions; however, it includes only the spectral contents and does not exhibit the frequency evolution with respect to time. Wavelets however have high time resolution for high frequency components and high frequency resolution for low frequency component [7]. The Short-Time Fourier transform (STFT), like wavelets, belongs to linear TimeFrequency Representations (TFRs). It is the most commonly used classical method for nonstationary signals' analysis. Its squared modulus produces the well-known spectrogram that is of the quadratic class. These two representations have been widely used due to its robustness and simplicity [8, 9]. However, their main drawback is that their overall performance strongly depends on the choice of two smoothing parameters that are not easy to select: the window's type and the window's length. Furthermore, according to the Heisenberg-Gabor uncertainty principle, there will be always a tradeoff between time and frequency resolutions. For the STFT, the use of too short analysis window yields to perfect time localization/bad frequency localization and the converse is true. This is in fact one of the fundamental practical arguments against the use of the STFT and the spectrogram [10]. The Wigner-Ville Distribution (WVD) is the oldest quadratic time-frequency transform; it offers one of the highest time and frequency resolutions of the signal original frequency components called autoterms. However, it generates high-energy interferences, called crossterms that degrade enormously its readability. Subsequently, some modifications have been proposed to improve the WVD performance, namely, the Smoothed-Pseudo-WignerVille Distribution (SPWVD) that is inspired from the main idea of separable smoothing windows in time and in frequency. These modifications have resulted in a remarkable suppression of crossterms, but to the detriment of the resolution $[10,11]$.

Cohen's class is the generalized form of quadratic time-frequency distributions that allows generation of a great variety of representations. Each of them depends on the mathematical expression of the related kernel. The latter smoothes the interferences that are systematically generated by the distribution itself [10]. However, from literature review, there is no specific distribution that is the best choice for all kinds of signals and applications [12-15]. This is because TFDs are signal-dependent and each one suffers from one or more limitation. Therefore, the ultimate challenge is to provide the best compromise between highest time-frequency resolution, highest signal's autoterms and greatest crossterms' elimination. Every classification process applied latter depends on these three criteria [16].

Our first contribution consists of analyzing the use of quadratic time-frequency representations of Cohen's class to provide a decision about the inverters' state. Among the available techniques, a recently proposed quadratic TFD, the PCBD, based on a polynomial kernel with compact support (KCS) is employed. A specific system is used to acquire real-life currents corresponding to the healthy and faulty cases. The performed diagnosis is based on instantaneous frequency estimation of current signals' components.

The second contribution consists of automatically select the optimal kernel parameter that results on the best resolution and interference suppression using the Stankovic concentration-based performance measure. The performed optimization is very fast because it is related to a single integer that adjusts the kernel's bandwidth. Furthermore, since the kernel vanishes itself outside a compact support, there is no need to external windows to improve time and/ or frequency resolution. Hence, the faults detection process is fully automatic.

The remainder of this paper is organized as follows. In Section 2, we describe the PCBD and its related timelag kernel. Section 3 introduces the Stankovic measure that is used to objectively select the optimal kernel smoothing parameter based on signal concentration. The proposed time-frequency-based method applied to two-level inverter systems faults' detection is described in Section 4 including the full currents' acquisition chain. Comparative experimental results between the PCBD and the most-known classical TFDs are provided in Section 5 giving details about open-circuit fault detection. Finally, Section 6 concludes the paper. 


\section{The Cohen's class- The PCB distribution (PCBD)}

Let $x_{a}(t)$ be the analytic signal associated to the real signal $x(t)$. The Instantaneous Autocorrelation Function IAF, denoted by , describes the distribution of signal power over frequencies, such that [11]:

$$
K_{x_{a}}(t, \tau)=x_{a}(t+\tau / 2) x_{a}^{*}(t-\tau / 2) \text {. }
$$

The IAF is a two-time variable function: the actual time $t$ and the lag $\tau$. The WVD of a signal $x(t)$ is defined as the Fourier transform of the time-dependent IAF [10]:

$$
\operatorname{WVD}_{x}(t, f)=\int_{-\infty}^{+\infty} x(t+\tau / 2) x^{*}(t-\tau / 2) e^{-2 j \pi f \tau} d \tau \text {. }
$$

It is important to note that the WVD results on the best time-frequency resolution and is perfect for monocomponent linear chirp signals [10]. However, its use is not practical for the reason explained below. Consider, as an example, a two-component nonstationary signal $x(t)=x_{1}(t)+x_{2}(t)$. Its WVD is expressed by [10]:

$$
\begin{aligned}
& \operatorname{WVD}_{s}(t, f)=\operatorname{WVD}_{s_{1}}(t, f)+\mathrm{WVD}_{s_{2}}(t, f) \\
& +2 \operatorname{Re} \int_{-\infty}^{+\infty} s_{1}(t+\tau / 2) s_{2}^{*}(t-\tau / 2) e^{-2 j \pi f \tau} d \tau .
\end{aligned}
$$

The last term in Eq. (3) represents interferences, crossterms or also called artifacts whose presence causes a significant degradation of the time-frequency plots' readability. This situation is illustrated in Fig. 1 presenting two non-parallel linear chirps while Fig. 2 shows the effect of introducing a smoothing window $h$ (Hamming, $L=55$ ) using the spectrogram [11]:

$$
S_{x}^{h}(t, f)=\left|\int_{-\infty}^{+\infty} x(\tau) h^{*}(t-\tau) e^{-j \pi f \tau} d \tau\right|^{2}
$$

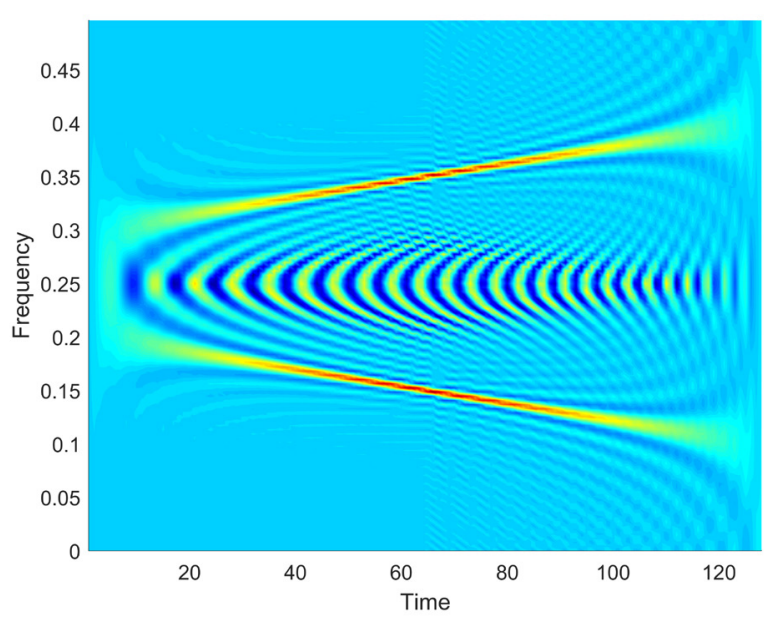

Fig. 1 The crossterms' structure of the WVD between two non-parallel linear chirps occupying the frequency ranges $f=[0.3-0.4] \mathrm{Hz}$ and $f=[0.2-0.1] \mathrm{Hz}$, respectively.

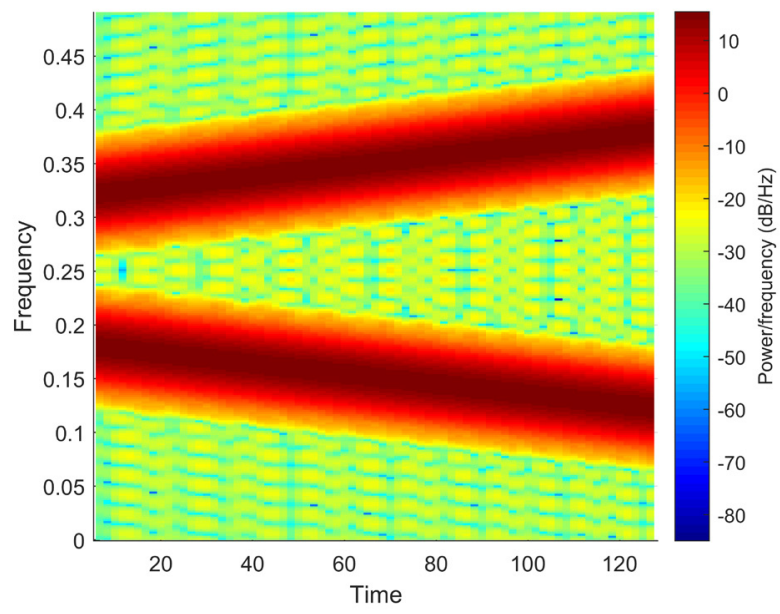

Fig. 2 Spectrogram of the signal of Fig. 1 using a Hamming analysis window of length $L=55$.

We see that the WVD provides very high autoterms' resolution while the crossterms are better suppressed using the spectrogram. However, the latter is recognized by its poor concentration that affects considerably the time-frequency localization (the start and the end frequencies of each component cannot be determined with precision from Fig. 2) It is desired to design joint $t-f$ representations that eliminate the best the artifacts generated by the WVD so that to extract the most precise energy information about the time-varying spectra [10].

In this paper, we deal with a specific class of quadratic distributions verifying time and frequency covariance, called the Cohen's class so that $[11,13,17]$ :

$$
\begin{aligned}
& \operatorname{TFD}_{x}(t, f) \\
& =\iiint_{-\infty}^{+\infty} e^{j 2 \pi \eta(s-t)} \phi(\eta, \tau) x\left(s+\frac{\tau}{2}\right) x^{*}\left(s-\frac{\tau}{2}\right) e^{-j 2 \pi f \tau} d \eta d s d,
\end{aligned}
$$

where $\phi(\eta, \tau)$ is the two-dimensional kernel whose role is to smooth interferences. Hence, the Cohen's class of distributions can be viewed as simply smoothed versions of the WVD, where the "smoothing" can be described in four plans. In this regard, using Fourier transforms from lag $\tau$ to frequency $f$ and from time $t$ to Doppler $\eta$, the quadratic TFDs can be expressed in four different but dependent two-dimensional domains namely the time-lag, the time-frequency, the Doppler-lag and the Dopplerfrequency domains [11]. Fig. 3 shows the possible transitions from the different kernels' domains.

Here, $F$ and $F^{-1}$ are the direct and inverse Fourier transforms. In particular, our formulation of the PCBD uses the time-lag notation $G(t, \tau)$ expressed as the Fourier transform of the Doppler-lag kernel $\varphi(\eta, \tau)$ with respect to $\eta[10,16]$ : 


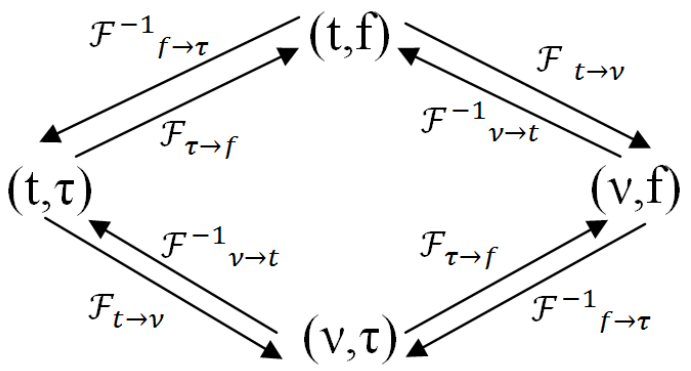

Fig. 3 Possible transitions from the four two-dimensional domains for quadratic TFDs [11].

$G(t, \tau)=\int_{-\infty}^{+\infty} \varphi(\eta, \tau) e^{-j 2 \pi \eta} d \eta$

Hence, the Cohen's class of distributions of an analytic signal $x_{a}(t)$ can be rewritten as follows [17]:

$\operatorname{TFD}_{x}(t, f)$

$=\int_{-\infty}^{+\infty} \int_{-\infty}^{+\infty} G(t-s, \tau) x_{a}\left(s+\frac{\tau}{2}\right) x_{a}^{*}\left(s-\frac{\tau}{2}\right) e^{-j 2 \pi f \tau} d s d \tau$.

This last expression is used in our numerical implementation of the PCBD since the number of integrals in reduced to two rather than three in Eq. (5). Furthermore, we deduce from Eq. (6) that any TFD of Cohen's class can be computed by convolution of its time-lag kernel $G$ with the analytic signal's IAF followed by Fourier transform. In order to achieve the best compromise mentioned above, a significant number of distributions have been proposed.

One of the most known distributions is Born-Jordan Distribution (BJD), where its kernel is defined as cardinal sine function. The main drawback of this distribution is that it does not contain any tuning parameter and hence it cannot be optimized. Furthermore, it generates positive and negative interferences especially for very close frequency components.

Choi-Williams Distribution (CWD) represents another well-known distribution whose Doppler-lag kernel is defined as a two-dimensional Gaussian function [14, 16]. The main drawback of the CWD is high crossterms' generation when the signal emerges two components located at the same moment or at the same frequency band [10].

Zhao-Atlas-Marks Distribution (ZAMD) offers an excellent autoterms resolution comparing to the previous distributions, but it is still limited in terms of reaching the perfect interferences' rejection, particularly the emergence of negative interferences near the signal's proper terms $[15,16]$.
The most recently used high-resolution distributions are the Modified-B Distribution (MBD) [18], the Extended MBD (EMBD) based on two smoothing parameters and the Compact Kernel Distribution (CKD) [19] that is tuned using three real parameters. In the same context, new promising tools derived from kernels with compact support (KCS) are considered [10, 12, 16, 20-22] so that the related kernel is adjusted using a single parameter. Inspired from the SPWVD, the approach of using separable kernel TFDs have recently gained more popularity. Among them, the EMBD and the SCB TFD proposed in [16, 22], such that an independent smoothing is performed along the frequency axis and the time axis, separately. Some recent TF representations based on S-transform combined with KCS are also investigated [23].

In this paper, we propose to use high-resolution time-frequency distribution based on a kernel with compact support. The latter, referred to as the PCB, is expressed in the time-lag domain as [16]:

$$
\begin{array}{ll}
G_{\mathrm{PCB}}(t, \tau)=\frac{\gamma+1}{\pi \lambda^{2 \gamma+2}}\left(\lambda^{2}-\left(t^{2}+\tau^{2}\right)\right)^{\gamma} & \text { if }\left(t^{2}+\tau^{2}\right)<\lambda^{2} \\
0 & \text { otherwise }
\end{array}
$$

where $\lambda$ is a predetermined fixed parameter and $\gamma$ is a positive integer that controls the kernel's bandwidth as $\sigma$ does for the Gaussian function. The resulting kernel is then of polynomial form. Furthermore, the $G_{\mathrm{PCB}}$ is of compact support which makes the PCBD computational cost very low and its optimization the easiest [16].

In order to appreciate the particularity of this kernel, it is useful to recall a very important property of time-frequency signal analysis. As depicted in Fig. 4, the ambiguity function's autoterms are located at the origin of the Doppler-delay plane whereas their crossterms are located away from the origin $[10,16]$. Hence, the Doppler-lag PCB (Fig. 5) obtained from inverse Fourier transform of $G_{\mathrm{PCB}}$ with respect to $t$ (see Eq. (6)) acts as a two-dimensional auto-windowed lowpass filter centered around the origin resulting on greater interferences' elimination while the signal proper terms are better preserved [16].

\section{Time-frequency representation's performance measurement}

An effective measure of the TFDs' concentration can provide a quantitative criterion for objective assessment of various distributions for a given test signal [24]. This may constitute an efficient tool for optimization of the kernel's 


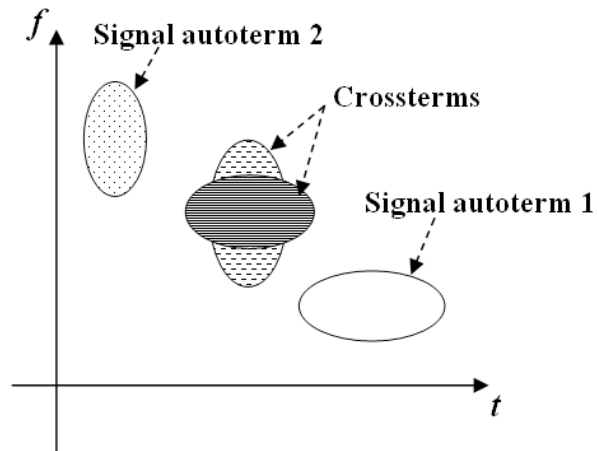

(a)

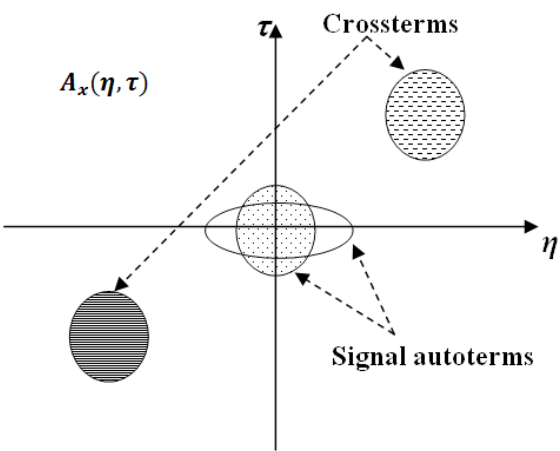

(b)

Fig. 4 Signal autoterms and interference terms location: (a) the time-frequency domain (the Wigner-Ville Distribution (WVD)) and (b) the Doppler-lag domain (the ambiguity function) [16].

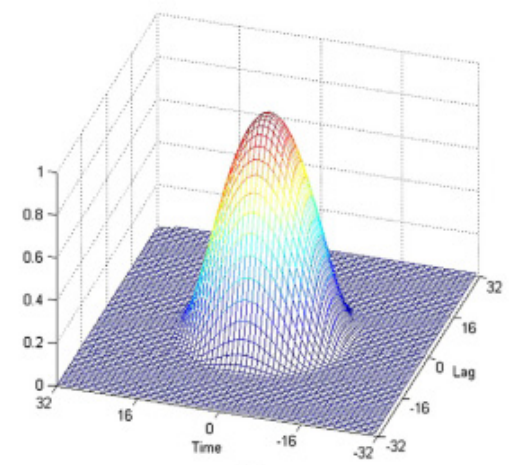

(a)

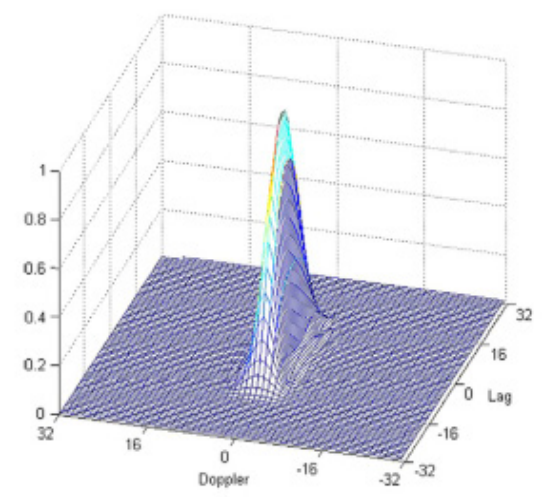

(b)

Fig. 5 The two-dimensional KCS profile for a fixed bandwidth $(\gamma=2.5, L=64)$. (a) the time-lag plane and (b) the Doppler-delay plane (the ambiguity domain) $[10,16]$. smoothing parameters, if they exist, and also automatic selection of the best performing TFD [16, 22, 25]. Among the few quantitative objective measures that are used in time-frequency signal analysis, it was shown in [16] through a deep comparative study that the Stankovic concentration-based performance measure defined as [25]:

$S_{\beta}=\left(\sum_{n} \sum_{k}\left|\operatorname{TFD}_{x}(n, k)\right|^{1 / \beta}\right)^{\beta}$,

provides the most accurate optimization results. Stankovic has mentioned in [25] that it is preferred to use a lower-order form to achieve the robustness, for example $\beta=4$. $S_{\beta}$ was used with the spectrogram to automatically select its optimal window's parameters while many researchers have increasingly used this measure in various applications with different optimized TFDs yielding to high autoterms' concentration and interferences' suppression [25-28]. Note that the optimal distribution from Stankovic measure point of view is the one that minimizes $S_{\beta}$.

\section{Time-frequency analysis requirement in inverter diagnosis}

Electric motors operating at constantly changing speeds and load conditions own voltages and currents whose frequency and amplitude are persistently changing over time, this describes the feature of nonstationary signals [7]. Actually, when the system is located in an industrial environment, the current signals' acquisition systems from Induction Motor (IMs) can undesirably cause a presence of relatively high noise levels. The most usual sources of noise during an acquisition process are definitely electromagnetic interference by dint of the running of other nearby machines; nonlinear loads, switching. On the other hand, inter-harmonics induced by inverters feeding the IM can strongly generate disturbances in the power line. These extra harmonics produced in the signal create frequency components that may have amplitude higher than the signal's autoterms' amplitude or the same [29]. Basically, spectrum analysis provides information about what frequencies are present in the signal, while Fourier transform presents an important handicap for signals with time-varying spectra. The situation becomes worse in the presence of noise. Efficient time-frequency analysis tools, and particularly quadratic TFDs of Cohen's class, permit providing a full description of the signal's characteristics including the number of components, the time durations and frequency bands over which these components are defined, the components' amplitudes and the Instantaneous Frequency (IF) laws [30]. In this regard, it was shown 
through several tests that the compact support kernels, in general, and the PCB time-lag kernel, in particular, outperform the other ones even for the hard case of closely spaced noisy multicomponent signals embedded in $0 \mathrm{~dB}$ additive white Gaussian noise [10, 16].

\subsection{Three-phase two-level inverter system}

The paper deals with-two level inverter feeding threephase motor induction. The structure of the inverter is illustrated in Fig. 6. Generally, the failures can constantly affect an inverter during its operational condition. As mentioned in Section 1, the most sensitive components of inverters are power equipments, more precisely the IGBTs due to their fragility. Two types of faults can be found:

1. Short-circuit fault remains the most severe fault. In the presence this kind of fault, the current extremely increases causing catastrophic breakdown as a fusion of its chip. The detection should take place rapidly in order to avoid the propagation of the fault to the whole inverter leg.

2. An open-circuit fault affecting the IGBT switches constituting the subject of the present work. The IGBT can be disconnected, damaged or a problem arises in its gate control signal. Under such a fault, the motor's performance degrades because of fluctuations in motor's speed and torque while the other two healthy legs introduce high values of current to hold the average torque and speed ensuring motor's continuous running. This makes this fault difficult to be quickly detected. Once this type of fault occurs, the start-up of the motor cannot always be guaranteed [31-33].

\subsection{The proposed TFD-based method for open-Circuit inverter faults detection}

Fig. 7 depicts the block diagram of the proposed TFDbased processing method. The inverter's current signals are obtained using a specific acquisition system that will be described latter. Particularly, and due to its simple

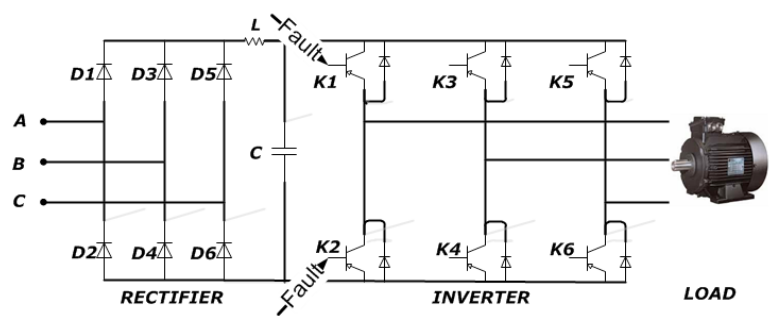

Fig. 6 Structure of the inverter-motor assembly under open-circuit fault [5].

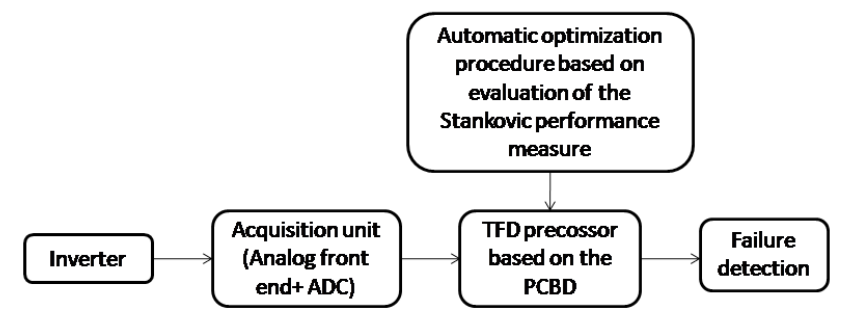

Fig. 7 Block diagram of the proposed processing method for opencircuit faults' detection.

tuning, the PCBD is employed together with unsupervised optimization procedure in order to detect the fault and localize the faulty arm online.

Consequently, the optimal kernel's parameter $\gamma$ is automatically adjusted using the Stankovic measure described in Section 3 yielding to the best signal proper terms resolution and artifacts suppression.

The flowchart presented in Fig. 8 details the main steps related to the proposed faults' detection method.

The acquisition chain of Fig. 9 [34] provides time-dependent currents since it is the most natural way of representing signals.

The TFD processor includes first a Hilbert transformer to produce the complex analytic signal that has by definition no negative components. Consequently, the bandwidth is reduced to the half compared to the real signal

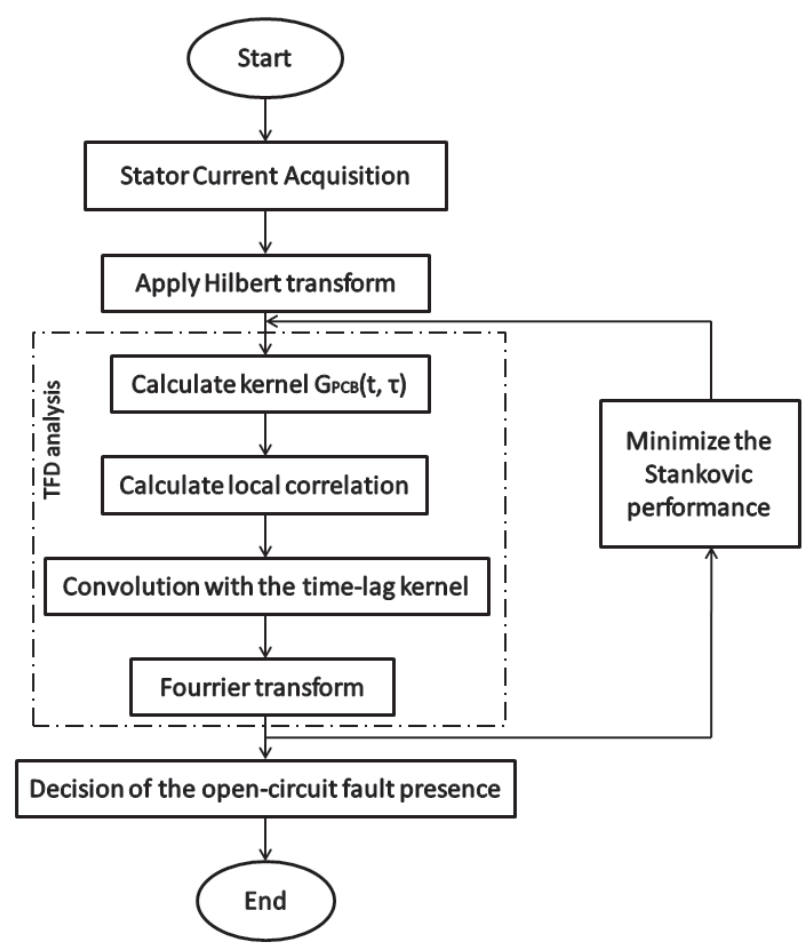

Fig. 8 Flowchart of the proposed unsupervised faults detection process based on the PCBD and the Stankovic measure. 


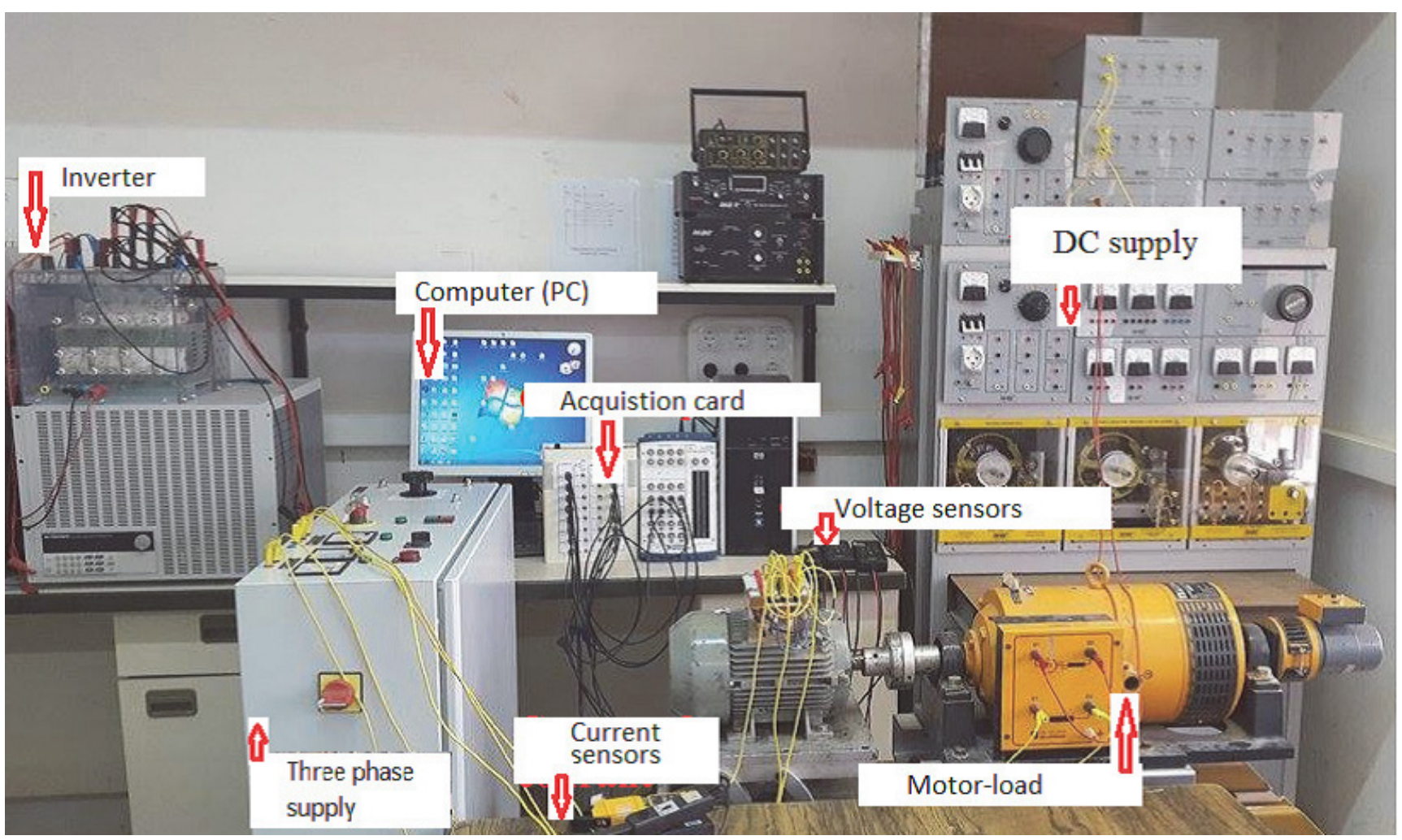

Fig. 9 Photo of the experimental test-rig [34].

$x(t)$ which means that the number of components in the time-frequency plane is also reduced to the half and then the spectral aliasing does not take place in the useful spectral domain of $x_{a}(t)$ [35]. Furthermore, the interaction between negative and positive frequency components is suppressed. The analytic signal is defined as:

$x_{a}(t)=T F^{-1}\{X(f)\} * T F^{-1}\{2 U(f)\}$

$=x(t) *\left[\delta(t)+j \frac{1}{\pi t}\right]$

$=x(t)+j\left[x(t) * \frac{1}{\pi t}\right]$

$x_{a}(t)=x(t)+j \hat{x}(t)$,

where $\hat{x}(t)$ is the Hilbert transform of the real signal $x(t)$.

The Hilbert transformer is followed by a local correlator to produce a plurality of signals corresponding to the delayed samples and combined by multiplication with their complex conjugates. The IAF associated to $x_{a}(t)$ is convloluted with the time-lag PCB kernel then followed by a Fourier transformer to generate the PCBD providing joint information in both time and frequency.

The three-phase inverter used in this work is an IGBTbased three-phase (SEMI-KRON) controlled by the DSPACE 1104 Card. The IGBTs are controlled by MLISVM. The motor used is of three-phase squirrel cage type; with a nominal power of $3 \mathrm{Kw}$, a frequency of $50 \mathrm{~Hz}$ and a nominal rotor speed of $1440 \mathrm{rpm}$. This motor is mechanically coupled to a DC generator used as a load. The measuring system has three voltage sensors (TEKTRONIX P5200) and three Hall-Effect current sensors (FLUCK i30s (AC/DC CURRENT CLAMP)), a tachometer (ONO SOKKI HT-341) and an acquisition card (NI-6330). Finally, the whole set is connected to a computer to display the processed acquired signals as shown in Fig. 9 [34].

\section{Experimental results}

Experimental tests are made on healthy and open-circuit faulty IGBT in order to show the effectiveness of the proposed time-frequency-based approach. The recorded current signals related to the healthy and faulty cases are depicted in Fig. 10 while the open-circuit faults are shown at different legs of the inverter. These temporal representations do not allow in any way the detection of failures.

Concerning the PCBD, Fig. 11 shows the effect of increasing the kernel's length $L$ on the behavior of the PCB in the ambiguity domain. It is clearly seen that as $L$ increases, the kernel becomes thinner and smoother so that it allows better crossterms' suppression while the signal's proper terms are maintained. This increases resolution of individual components. However, as it is the 


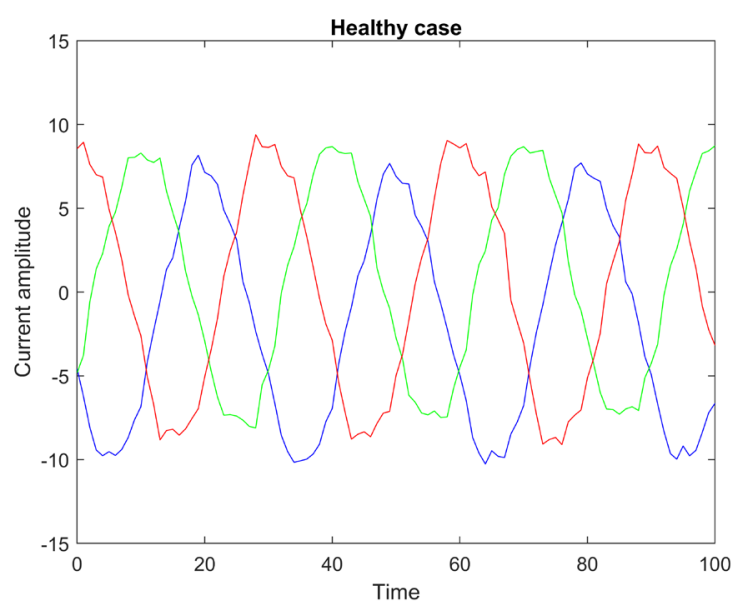

(a)

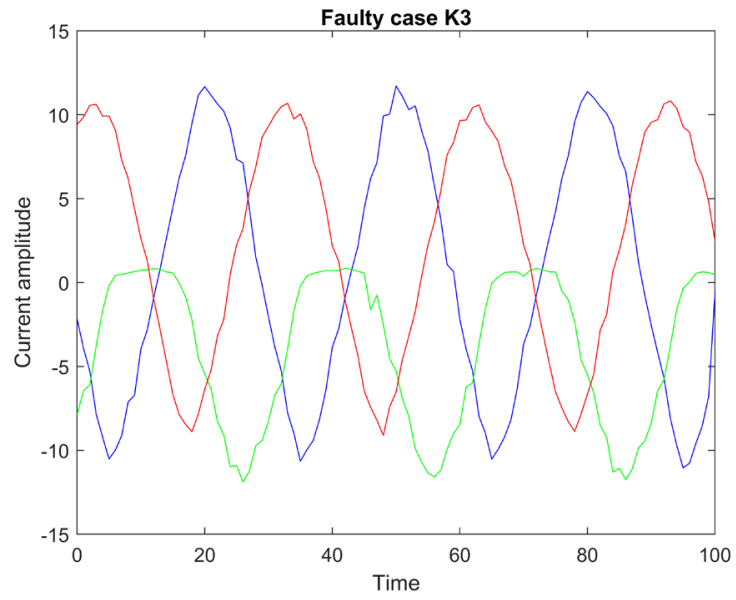

(c)

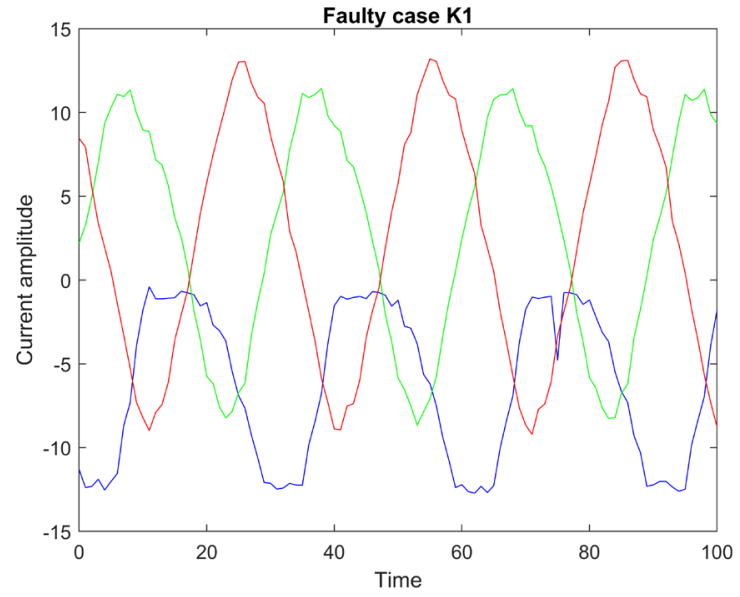

(b)

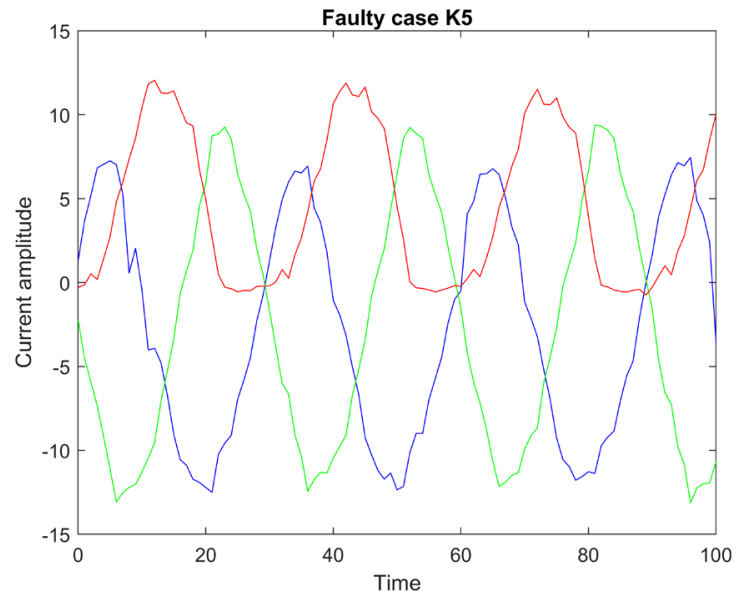

(d)

Fig. 10 Temporal representation of current signals for healthy and faulty cases at different inverter's legs: (a) healthy case; (b) faulty open-circuit K1 case; (c) faulty open-circuit K3 case; (d) faulty open-circuit K5 case.

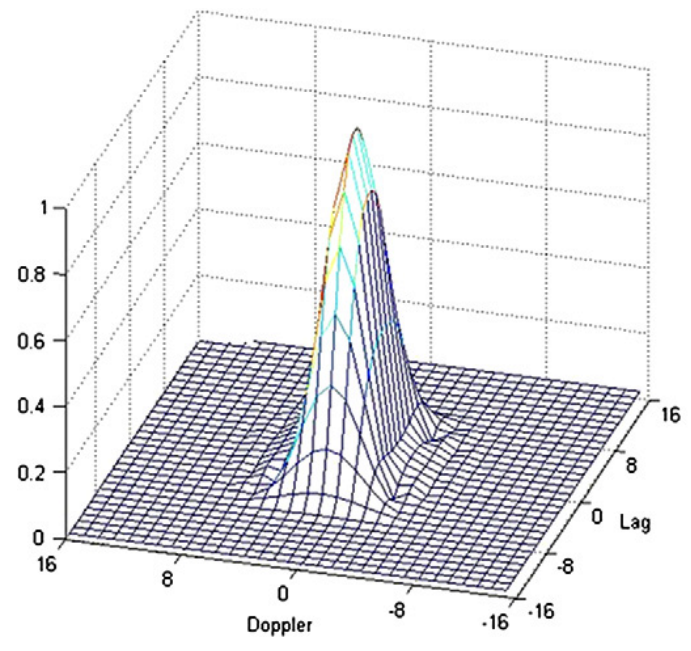

(a)

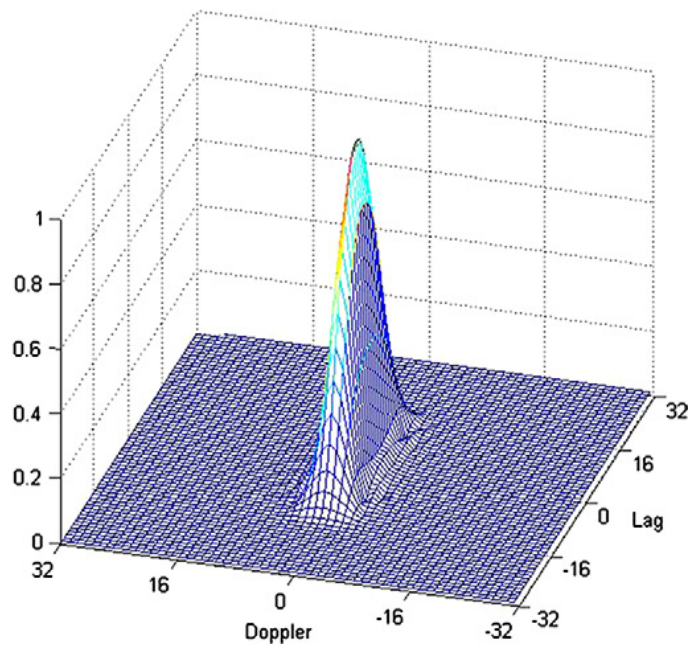

(b)

Fig. 11 The two-dimensional KCS profile in the ambiguity domain normalized with respect to its peak for a fixed bandwidth $(\gamma=2.5)$. (a) $L=32$ and (b) $L=64$ [21]. 
case of all filters, smoothing decreases concentration. This degrades time-frequency localization, i.e. the TFD's ability to exactly determine the appearance and disappearance instants related to each frequency event. This is not important in our case since the main objective is to avoid:

- First, appearance of crossterms that can be interpreted as open-circuit inverter faults while the motor is healthy and,

- second, appearance of other frequency components related to other faults' types. In the two cases, the result is a bad diagnosis. In what follows, the kernel's length $L$ is set to 512 . Whereas $\gamma$ is optimally defined through minimizing the Stankovic concentration-based performance measure given by Eq. (9). For the purpose of the present work, $\beta$ is set to 4 that corresponds to the smallest integer giving useful information measure for this type of signals.

First, we consider the signal acquired in the healthy case of the inverter. Fig. 12 presents $S_{4}$ for different values of the PCBD's tuning parameter $\gamma$. From the plot, we clearly see that $S_{4}$ is a convex function and hence it possesses certainly a global minimum that is in this case located at $\gamma=3$.The PCBD is thus auto-tuned at this optimal parameter. Let us now analyze the stator current in the time-frequency domain considering both the healthy and the open-circuit faulty IGBT.

Fig. 13 tells us about the fundamental harmonic of the current inverter under healthy conditions that corresponds to the frequency component $50 \mathrm{~Hz}$. On the other hand, by inspection of the PCBD first harmonics for the healthy and faulty cases, we see that open-circuit faults detection lies on the presence of zero-order harmonics that

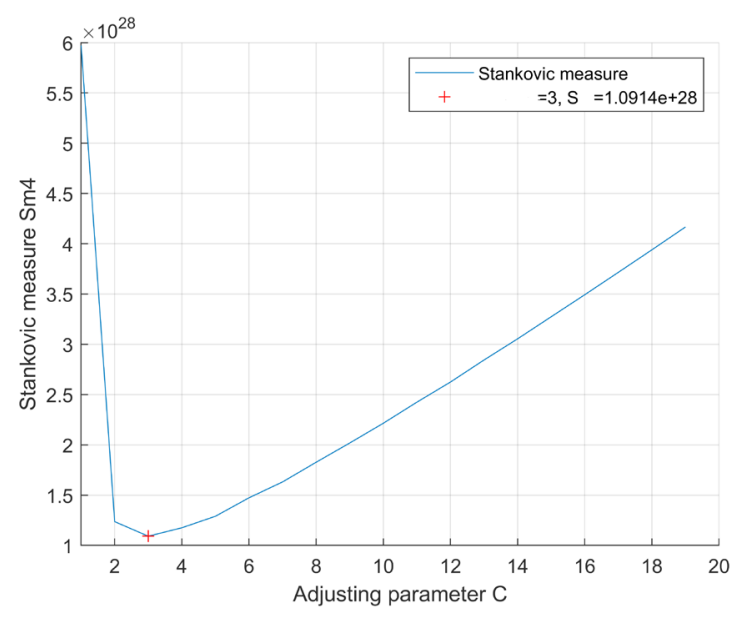

Fig. 12 Stankovic measure of the PCBD at different value of $\gamma$.

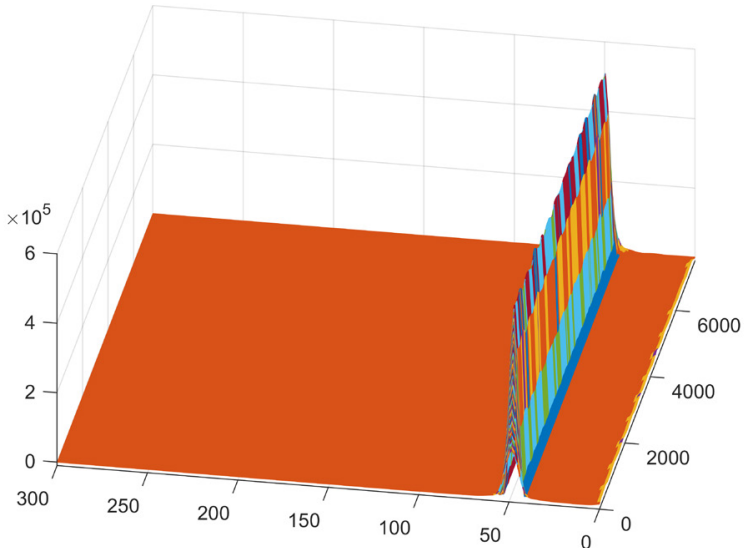

(a)

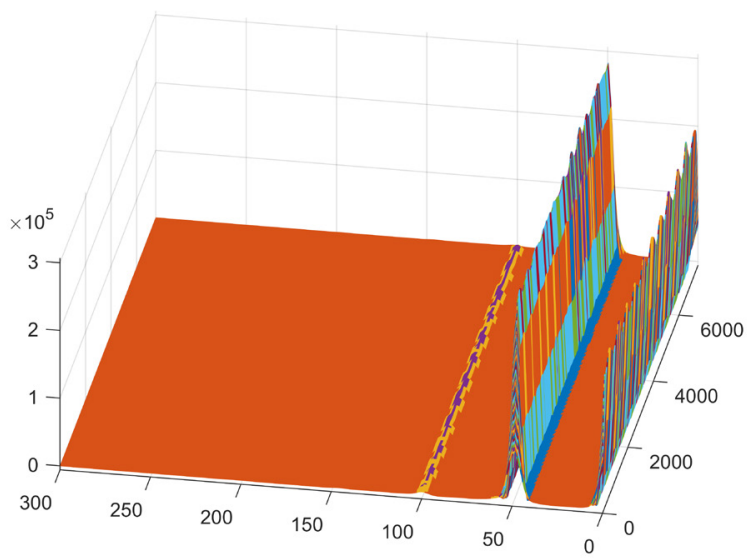

(b)

Fig. 13 The PCBD of the inverter's current signal: (a) healthy case $(\gamma=3)$ and (b) open-circuit faulty case at $\mathrm{K} 1(\gamma=3)$.

affirm the presence of a DC component in the current signal. The appearance of a zero harmonic in addition to the fundamental harmonic does not allow us to determine exactly the type of fault neither to know the faulty switch (the upper one or the lower one). This is due to the fact that DC components appear in all phases when the fault.

From Fig. 13 (b), we see that the existence of an open-circuit switch fault at K1 generates two important frequency components at $0 \mathrm{~Hz}$ and $100 \mathrm{~Hz}$ while analyzing the current signal of the corresponding phase A in the TF plane. An open-circuit fault is thus detected by the presence of the frequency component $100 \mathrm{~Hz}$ that represents the double of the fundamental frequency component $50 \mathrm{~Hz}$.

An open-circuit fault is thus detected by the presence of the frequency component $100 \mathrm{~Hz}$ that represents the double of the fundamental frequency component $50 \mathrm{~Hz}$.

In order to provide a comparative study, let us analyze the time-frequency characteristics of the faulty inverter's 
state using a selection of the best known TFDs as depicted in Fig. 14. The adjusting parameters related to each TFD are the optimal values corresponding to the minimum achieved Stankovic measure except for the WVD and the BJD that have no smoothing parameters and hence they cannot be optimized. We observe that the current signal's proper terms are better discriminated and concentrated around their respective bandwidths using the PCBD with $\gamma=3$ followed by the EMBD that performs separable smoothing in time and frequency with smoothing parameters $\alpha=0.001$ and $\beta=0.9$. However, the tuning of the PCB kernel is much more flexible and easier than the EMBD's kernel that needs two real parameters to be correctly adjusted.

The WVD results on high autoterms' resolution to the detriment of crossterms' generation. This causes the appearance of extra frequency components that do not among to the signal itself and hence provide false diagnosis. On the other hand, the appearance of inner and outer interferences as supplementary fluctuations in the TF diagrams reduces the overall performance of the BJD, the CWD and the ZAMD. The latter exhibits high negative crossterms' amplitudes near the signal's autoterms.

The spectrogram with the used setting shows high interferences that prevent to discriminate the signal's components and provide false diagnosis. This constitutes the major drawback of this representation that strongly depends on the selection of the right window's type and width which is not obvious.

In order to examine deeply the resolution performance, we inspect in Fig. 15 the time slices plots nearly the middle of the signal duration $t_{0}=3500 \mathrm{sec}$. We see that the PCBD provides high concentration of the autoterms around their IF laws and reduces the best the crossterms besides while adjusted and optimized using a single integer parameter $\gamma=3$. The resolution performance of the EMBD is superior to the other remaining distributions. However, two drawbacks are encountered: First, the kernel requires the tuning of two real parameters and second, resolution improvement needs the use of two external windows that smooth interferences in time and frequency, respectively. This makes the EMBD's setting more complicated because there are too many parameters to adjust: the kernel's smoothing parameters, the windows' types and their respective lengths. The ZAMD, the BJD and the CWD distributions show the highest resolution of the signal components but induce other harmonics of high negative amplitudes especially in the close neighborhood of the autoterms. Similarly, the WVD suffers from the crossterms problem and generates extra interference terms that have amplitudes higher than the signal's proper terms. This situation is clearly seen at the frequency bands $[0,50] \mathrm{Hz}$ and $[50,100] \mathrm{Hz}$. As a result, the readability of the TF diagrams is severely affected which may falsify the performed diagnosis. Concerning the spectrogram, the main drawbacks are bad resolution due to poor concentration of the signal's proper terms and also the fact that its performance in reducing interferences highly depends on the choice of the window's type and width.

Fig. 15 (h) reports the time slices obtained from the PCBD for different values of $\gamma$. The purpose is to prove the efficiency of using the Stankovic performance measure for automatic tuning of the distribution. We see that when $\gamma=3$, the PCBD achieves the best tradeoff between high resolution and interference rejection. However, as $\gamma$ decreases below this optimal value, negative crossterms appear. On the other hand, as $\gamma$ increases, the instantaneous bandwidths centered about the components' IFs become larger. This causes losses in conservation of the autoterms' concentration due to the smoothing effects of the compact support kernel.

\section{Conclusion}

Automatic detection of open-circuit faults in inverters is particularly illustrated in this paper. The analyzed real-life currents are provided from a test bench at LDEE laboratory.

The proposed method consists of using a recently proposed time-frequency distribution, the PCBD, together with an optimization process based on the Stankovic measure for automatic parameterization of the kernel that has a polynomial form.

The obtained results show the superiority of the PCBD against the most known TFDs that remain either limited in ensuring the best resolution and minimizing interferences or difficult to adjust. In this context, the PCBD offers many substantial advantages: First, the kernel's tuning is done using a single integer parameter which makes the distribution's setting and optimization the easiest.

Second, there is no need to external windows to smooth the automatically generated crossterms while preserving the signal proper terms since the window is the kernel itself.

Third, the computational cost of the distribution is the lowest because it vanishes outside a compact support. This reduces considerably the number of points that need computation.

We have also proved the efficiency of using the Stankovic concentration-based performance measure for automatic selection of the optimal representation. 


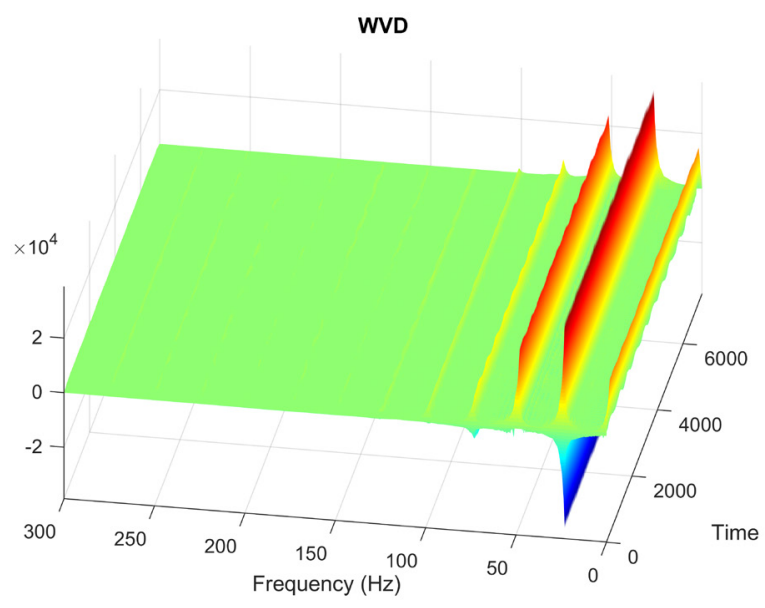

(a)

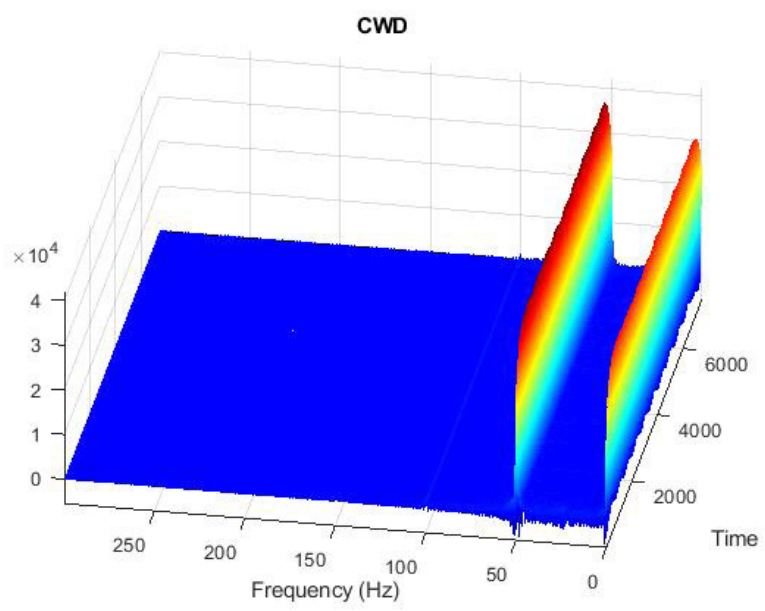

(c)

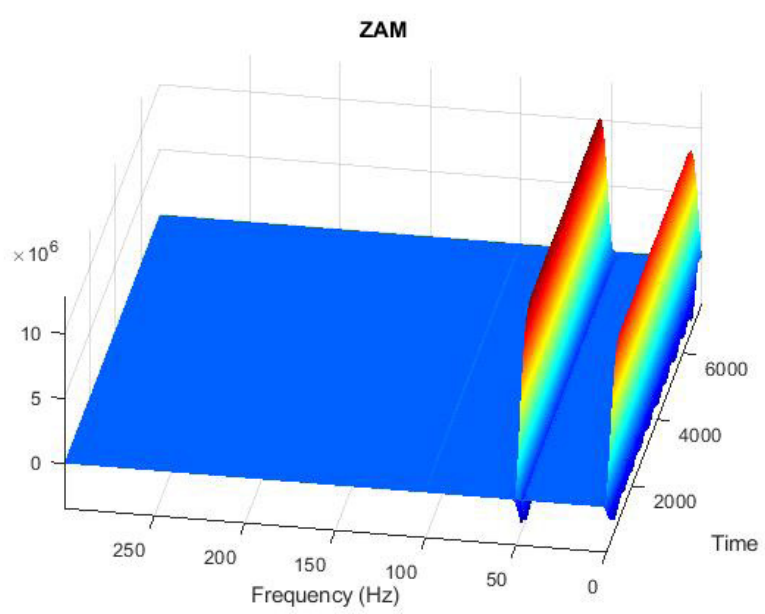

(e)

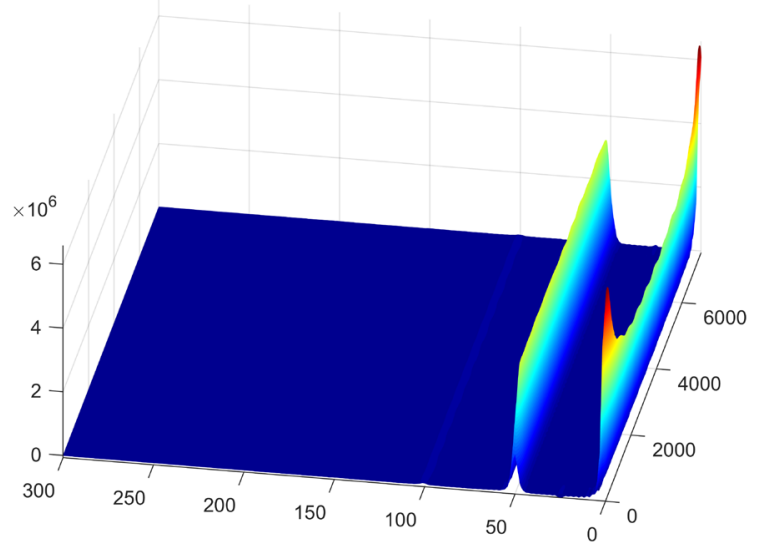

(b)

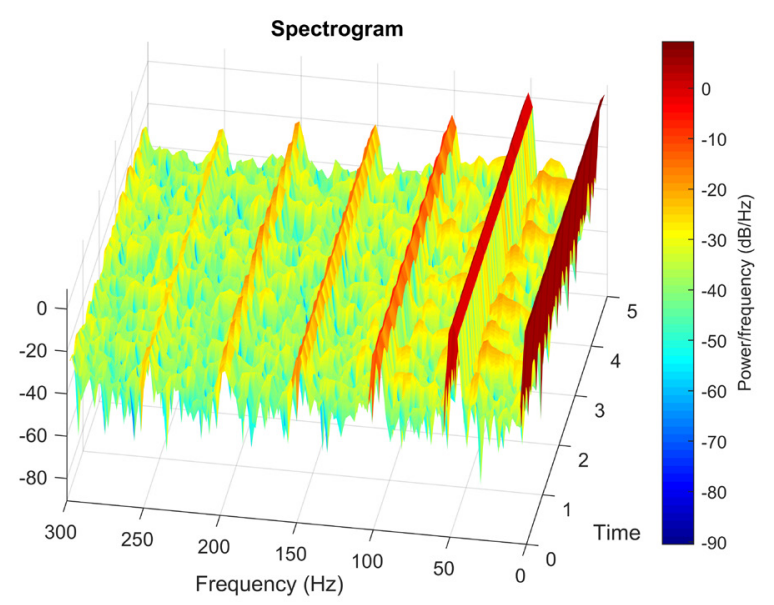

(d)

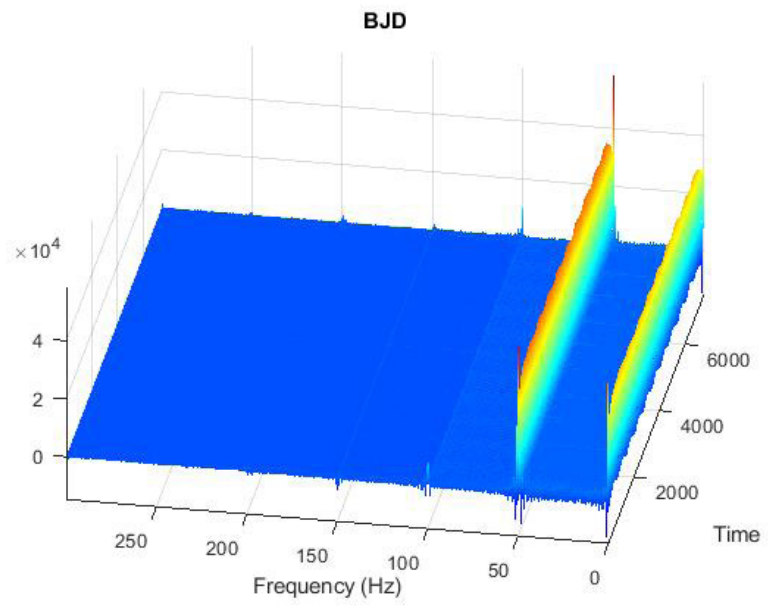

(f)

Fig. 14 TFDs of faulty open-circuit $K 1$ signal: (a) WVD; (b) EMBD ( $\alpha=0.001, \beta=0.9$ ); (c) CWD ( $\beta=1)$; (d) Spectrogram (Hamming, $L=512$ ); (e) ZAMD ( $\alpha=1)$; (f) BJD

In fact, it must be noted that a distribution that is not properly adjusted leads to a false diagnosis. This is due to bad estimation of the true time-varying signal components. Concerning open-circuit faulty IGBT, the stator current signal is found to be characterized by a DC component indicating the presence of a fault and a $100 \mathrm{~Hz}$ frequency component corresponding to the open-circuit fault. The proposed time-frequency-based method can 


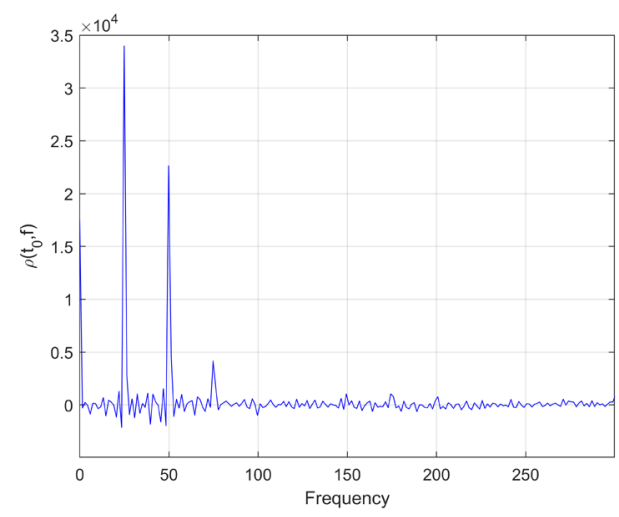

(a)

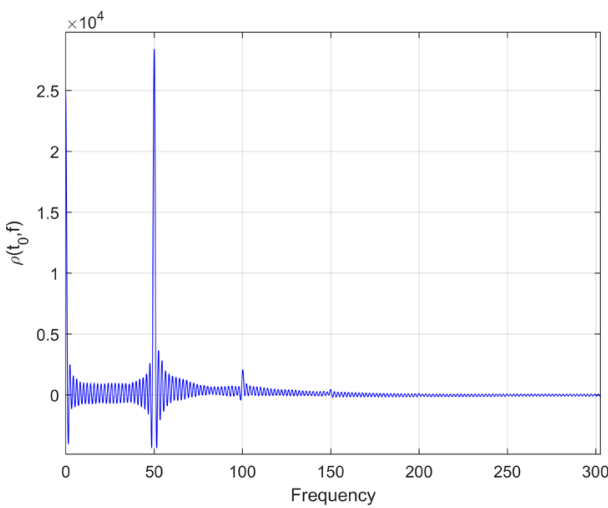

(c)

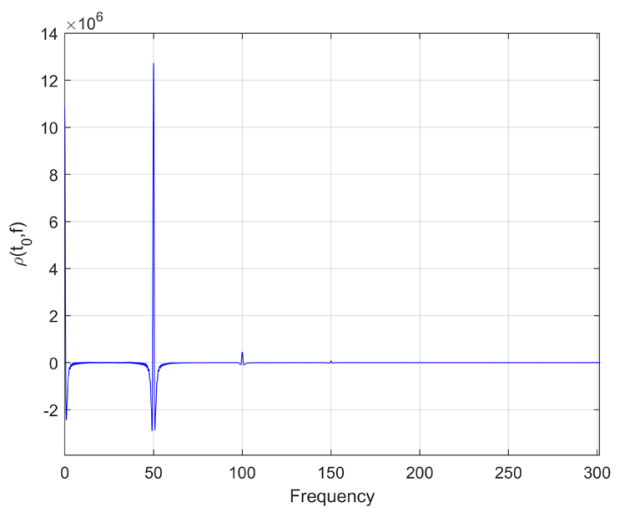

(e)

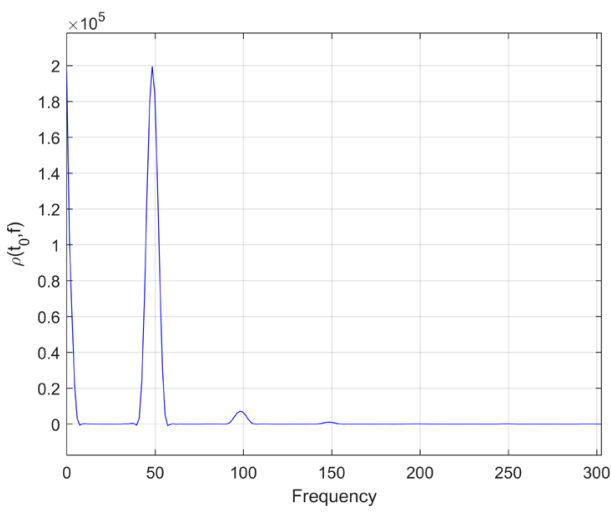

(g)

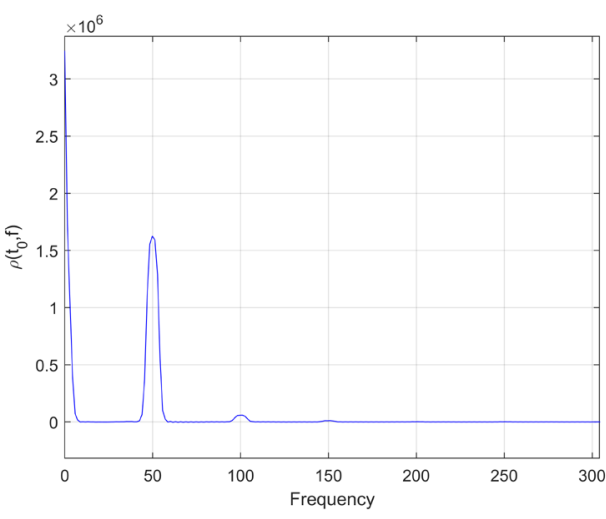

(b)

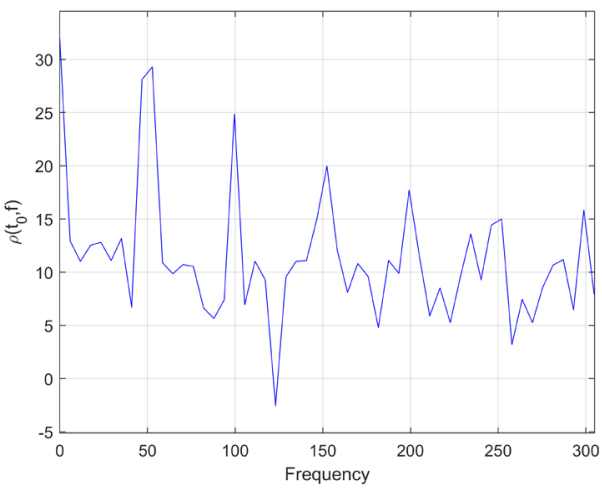

(d)

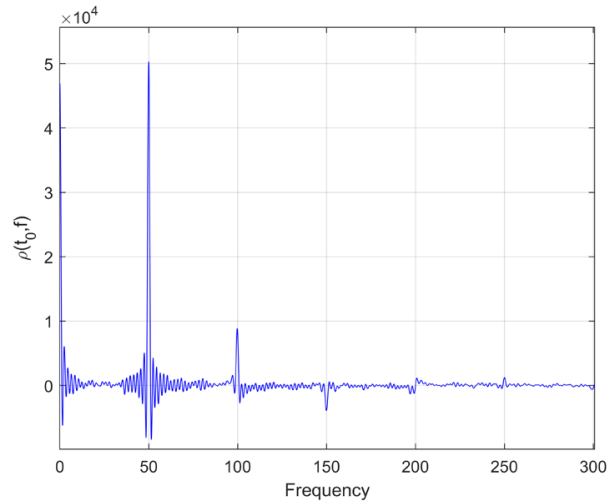

(f)

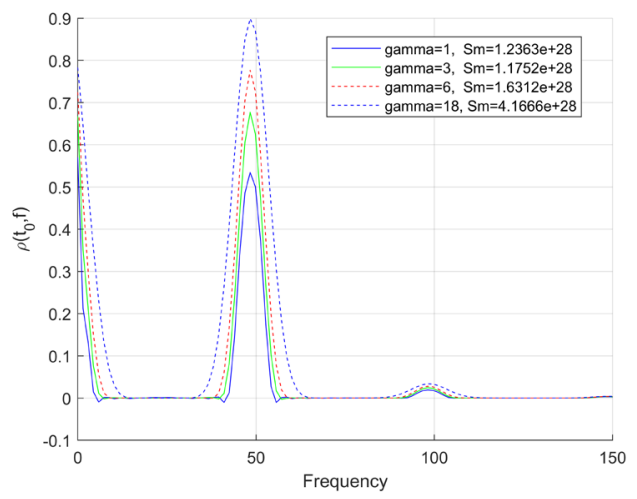

(h)

Fig. 15 Time Slice of a TDF $\rho(t, f)$ of faulty open-circuit K1 signal taken at the time instant 3500 sec: (a) WVD; (b) EMBD ( $\alpha=0.001, \beta=0.9)$; (c) CWD $(\beta=1)$; (d) Spectrogram (Hamming, $L=512$ ); (e) ZAMD $(\alpha=1)$; (f) BJD; (g) PCBD $(\gamma=3$ ); (h) PCBD for different values of $\gamma$. 
be extended to detect other faults such as bearing faults and broken rotor bars in induction motors. Furthermore, the implementation of the proposed technique to the drive

\section{References}

[1] Abdellah, C., Miloud, H., Azeddine, B., Pusca, R., Romary, R., Lecointe, J. P. "Instantaneous Power Spectrum Analysis To Detect Mixed Eccentricity Fault In Saturated Squirrel Cage Induction Motor", In: 3rd International Conference on Automation, Control, Engineering and Computer Science (ACECS-2016), Hammamet, Tunisia, 2016, pp. 296-300.

[2] Cherif, B. D. E., Bendiabdellah, A., Bendjebbar, M. "Detection of a two-level inverter open-circuit fault using the discrete wavelet transforms technique", In: 2018 IEEE International Conference on Industrial Technology (ICIT), Lyon, France, 2018, pp. 370-376. https://doi.org/10.1109/ICIT.2018.8352206

[3] Lu, B., Sharma, S. K. "A Literature Review of IGBT Fault Diagnostic and Protection Methods for Power Inverters", IEEE Transactions on Industry Applications, 45(5), pp. 1770-1777, 2009. https://doi.org/10.1109/TIA.2009.2027535

[4] Estima, J. O., Marques Cardoso, A. J. "A New Algorithm for RealTime Multiple Open-Circuit Fault Diagnosis in Voltage-Fed PWM Motor Drives by the Reference Current Errors", IEEE Transactions on Industrial Electronics, 60(8), pp. 3496-3505, 2012.

https://doi.org/10.1109/TIE.2012.2188877

[5] Cherif, B. D. E., Bendiabdellah, A., Bendjebbar, M., Telli, A. "A comparative study between methods of detection and localisation of open-circuit faults in a three phase voltage inverter fed induction motor", International Journal of Modelling, Identification and Control (IJMIC), 29(4), pp. 327-340, 2019. https://doi.org/10.1504/IJMIC.2018.10013129

[6] Trabelsi, M., Boussak, M., Gossa, M. "Multiple IGBTs open circuit faults diagnosis in voltage source inverter fed induction motor using modified slope method", In: The XIX International Conference on Electrical Machines-ICEM 2010, Rome, Italy, 2010, pp. 1-6. https://doi.org/10.1109/ICELMACH.2010.5608044

[7] Ahmad, N. S., Abdullah, A. R., Bahari, N., Hassan, M. A. A. "Switches Faults Analysis of Voltage Source Inverter (VSI) using Short Time Fourier Transform (STFT)", International Review on Modelling and Simulation, 7(3), pp. 409-415, 2014. https://doi.org/10.15866/iremos.v7i3.2183

[8] Rajagopalan, S., Restrepo, J. A., Aller, J. M., Habetler, T. G., Harley, R. G. "Nonstationary Motor Fault Detection Using Recent Quadratic Time-Frequency Representations", IEEE Transactions on Industry Applications, 44(3), pp. 735-744, 2008.

https://doi.org/10.1109/TIA.2008.921431

[9] Cherif, B. D. E., Bendiabdellah, A., Bendjebbar, M. "Experimental Study of Inverter Open-Circuit Fault Diagnosis using Stator Current Spectrogram", In: 5th International Conference on Automation, Control Engineering and Computer Science (ACECS 2018), Hammamet, Tunisia, 2018, pp. 1-5.

[10] Abed, M. "Time-Frequency Distributions: Compact Support Kernels, Performances and Implementations", PhD Thesis, Ecole Nationale Polytechnique, 2015. microcontroller/processor constitutes the object of our current work that will be presented in our future publications.

[11] Boashash, B. "Time-Frequency Signal Analysis and Processing: A Comprehensive Reference", Academic Press, London, UK, 2015.

[12] Belouchrani, A., Cheriet, M. "On the use of a new compact support kernel in time frequency analysis", In: Proceedings of the 11th IEEE Signal Processing Workshop on Statistical Signal Processing (Cat. No. 01TH8563), Singapore, Singapore, 2001, pp. 333-336. https://doi.org/10.1109/SSP.2001.955290

[13] Boashash, B. "Time-Frequency Signal Analysis and Processing: A Comprehensive Reference", Elsevier, Amsterdam, Netherlands, 2003.

[14] Choi, H. I., Williams, W. J. "Improved time-frequency representation of multicomponent signals using exponential kernels", IEEE Transactions on Acoustics, Speech, and Signal Processing, 37(6), pp. 862-871, 1989. https://doi.org/10.1109/ASSP.1989.28057

[15] Zhao, Y., Atlas, L. E., Marks, R. J. "The use of cone-shaped kernels for generalized time-frequency representations of nonstationary signals", IEEE Transactions on Acoustics, Speech, and Signal Processing, 38(7), pp. 1084-1091, 1990. https://doi.org/10.1109/29.57537

[16] Abed, M., Belouchrani, A. "Performance analysis and computational cost evaluation of high-resolution time-frequency distributions derived from compact support time-lag kernels", Digital Signal Processing, 78, pp. 1-19, 2018.

https://doi.org/10.1016/j.dsp.2018.02.017

[17] Cohen, L. "Time-frequency analysis", Prentice Hall, Englewood Cliffs, NJ, USA, 1995.

[18] Barkat, B., Boashash, B. "A high-resolution quadratic time-frequency distribution for multicomponent signals analysis", IEEE Transactions on Signal Processing, 49(10), pp. 2232-2239, 2001. https://doi.org/10.1109/78.950779

[19] Boashash, B., Khan, N. A., Ben-Jabeur, T. "Time-frequency features for pattern recognition using high-resolution TFDs: A tutorial review", Digital Signal Processing, 40, pp. 1-30, 2015. https://doi.org/10.1016/j.dsp.2014.12.015

[20] Cheriet M., Belouchrani A. "Method and system for measuring the energy of a signal", World Intellectual Property Organization, USA, Patent number: WO02088760 A2, 2002.

[21] Abed, M., Belouchrani, A., Cheriet, M., Boashash, B. "Compact support kernels based time-frequency distributions: Performance evaluation", In: 2011 IEEE International Conference on Acoustics, Speech and Signal Processing (ICASSP), Prague, Czech Republic, 2011, pp. 4180-4183. https://doi.org/10.1109/ICASSP.2011.5947274

[22] Abed, M., Belouchrani, A., Cheriet, M., Boashash, B. "TimeFrequency Distributions Based on Compact Support Kernels: Properties and Performance Evaluation", IEEE Transactions on Signal Processing, 60(6), pp. 2814-2827, 2012. https://doi.org/10.1109/TSP.2012.2190065 
[23] Zidelmal, Z., Hamil, H., Moukadem, A., Amirou, A., OuldAbdeslam, D. "S-transform based on compact support kernel", Digital Signal Processing, 62, pp. 137-149, 2017. https://doi.org/10.1016/j.dsp.2016.11.008

[24] Boashash, B., Reilly, A. P. "Algorithms for time-frequency signal analysis", Longman Cheshire, Melbourne, Australia, 1992.

[25] Stanković, L. "A measure of some time-frequency distributions concentration", Signal Processing, 81(3), pp. 621-631, 2001. https://doi.org/10.1016/S0165-1684(00)00236-X

[26] Brajović, M., Orović, I., Daković, M., Stanković, S. "On the parameterization of Hermite transform with application to the compression of QRS complexes", Signal Processing, 131, pp. 113-119, 2017. https://doi.org/10.1016/j.sigpro.2016.08.007

[27] Boashash, B., Ouelha, S. "Automatic signal abnormality detection using time-frequency features and machine learning: A newborn EEG seizure case study", Knowledge-Based Systems, 106, pp. 38-50, 2016.

https://doi.org/10.1016/j.knosys.2016.05.027

[28] Meignen, S., Pham, D. H., McLaughlin, S. "On Demodulation, Ridge Detection, and Synchrosqueezing for Multicomponent Signals", IEEE Transactions on Signal Processing, 65(8), pp. 2093-2103, 2017. https://doi.org/10.1109/TSP.2017.2656838

[29] de Jesus Romero-Troncoso, R. "Multirate Signal Processing to Improve FFT-Based Analysis for Detecting Faults in Induction Motors", IEEE Transactions on Industrial Informatics, 13(3), pp. 1291-1300, 2017. https://doi.org/10.1109/TII.2016.2603968
[30] Boashash, B., Sucic, V. "Resolution measure criteria for the objective assessment of the performance of quadratic time-frequency distributions", IEEE Transactions on Signal Processing, 51(5), pp. 1253-1263, 2003. https://doi.org/10.1109/TSP.2003.810300

[31] Singh, G. K. "Multi-phase induction machine drive researcha survey", Electric Power Systems Research, 61(2), pp. 139-147, 2002.

https://doi.org/10.1016/S0378-7796(02)00007-X

[32] Cherif, B. D. E., Bendiabdellah, A. "Detection of Two-Level Inverter Open-Circuit Fault Using a Combined DWT-NN Approach", Journal of Control Science and Engineering, 2018, Article ID: 1976836, 2018. https://doi.org/10.1155/2018/1976836

[33] An, Q. T., Sun, L. Z., Zhao, K., Sun, L. "Switching Function ModelBased Fast-Diagnostic Method of Open-Switch Faults in Inverters Without Sensors", IEEE Transactions on Power Electronics, 26(1), pp. 119-126, 2011. https://doi.org/10.1109/TPEL.2010.2052472

[34] Cherif, B. D. E., Bendiabdellah, A., Tabbakh, M. "Diagnosis of an Inverter IGBT Open-circuit Fault by Hilbert-Huang Transform Application", Traitement du Signal, 36(2), pp. 127-132, 2019. https://doi.org/10.18280/ts.360201

[35] Auger, F., Flandrin, P., Gonçalvès, P., Lemoine, O. "Timefrequency toolbox", CNRS, Paris, France, Rice University, Texas, USA, 1996. 